The book is stated to require no more than an advanced school knowledge of physics, and this accounts for explanatory notes on, for example, the Bohr atom and kinetic theory. There are good bibliographies with suggestions for further reading at the end of every chapter, and exercises with answers to numerical problems are provided.

$$
\text { U. W. Arndi }
$$

\section{Electricity and Magnetism}

Classical and Modern. By Dr. G. B. Deodhar and K. S. Singwi. Pp. viii +507. (Allahabad and Benares : Students' Friends, 1949.) 15 rupees.

7 HE authors, who are members of the staff of the Physics Department of the University of Allahabad, have covered the main topics in the subjects of electricity and magnetism at a level which is adequate for a pass degree in physics. Some of the material included, such as Maxwell's electromagnetic equations, goes beyond the usual range of a pass degree course, but generally the treatment is neither sufficiently advanced nor sufficiently comprehensive to meet the full needs of an honours course in physics.

The book is divided into two sections, classical and modern. The classical section covers the usual field, particular prominence being given to measuring instruments and their uses. The modern section outlines the subjects of the conduction of electricity through gases, electronics, positive ray analysis and isotopes, atomic structure, $\mathrm{X}$-rays, photoelectricity, natural radioactivity, artificial transmutation of elements, electron waves, cosmic rays, terrestrial magnetism and atmospheric electricity.

The book is lucidly written and well illustrated, and because emphasis is laid on experiments and physical concepts it should appeal to students with limited mathematical attainments. The quality of the printing and the trimming of the pages leave much to be desired.

A. $\mathrm{H}$.

Die elektromagnetische Schirmung in der Fernmelde- und Hochfrequenztechnik

Von Dr. Heinrich Kaden. (Technische Physik in Einzeldarstellungen, herausgegeben von W. Meissner, Band 10.) Pp. viii + 274. (Berlin, Göttingen und Heidelberg: Springer-Verlag; München : J. F. Bergmann, 1950.) 38 D. marks.

$A \mathrm{~S}$ is justly remarked in the preface, no collected A and systematic presentation of the principles of electromagnetic screening has hitherto been available. The author, with his long experience in these problems at Siemens-Halske, has been successful in filling the gap with this present book. Though the approach is essentially theoretical, it is concise and thorough and contains material, much of which is new, and most of which will prove valuable to the communications engineer who is concerned with screening at audio and radio frequencies.

The first part, "Screening against Disturbing Fields", which lays somewhat more emphasis on magnetic than on electric screening, deals with both closed and open screens and with the effect of slits, holes and gaps. Screening by grids and meshes of wires is only rather briefly treated. 'The second part, "Screening against Disturbing Currents", is concerned with undesired pick-up on the inner conductors of a cable due to imperfections of conductivity or continuity in the outer screen and with cross-talk between cables.

There is a short appendix which summarizes the properties of Bessel and Legendre functions and gives a selected bibliography of forty-nine references. A moderate level of mathematical knowledge in the reader is assumed, but the author has been careful to introduce and develop the techniques in a manner which is readily applied to similar problems having different boundary conditions. R. E. BuRGEss

\section{L'Analysis situs et la géométrie algébrique}

Par S. Lefschetz. (Collection de monographies sur la théorie des fonctions.) Pp. vi+154. (Paris: Libr. Gauthier-Villars, 1950.) 650 francs.

7 HIS reprint restores to currency one of the most 1 famous of the Borel tracts. The study of algebraic functions of a single variable rested securely on the pillars of function theory and topology, but the extensions and generalizations to higher dimensions were unsatisfactory until Lefschetz took the whole matter in hand; his investigations up to 1924 are summed up in this volume - not altogether easy read. ing and in places displaying some of the roughness of pioneering work, but full of ideas and brilliantly stimulating. The central position of topological notions in the field of algebraic geometry is clearly brought out in studying algebraic surfaces, systems of curves on algebraic surfaces, algebraic varieties, and the related Abelian integrals. From this guiding clue much of the very considerable progress made during the past twenty-five years in the domain of algebraic geometry has arisen. An account of this progress is to be found in Prof. W. V. D. Hodge's presidential address to the London Mathematical Society in $1949(J$. Lond. Math. Soc., 25, Part 3; July 1950), in which the great debt owed to Lefschetz by all algebraic geometers is fully acknowledged. 'The impact of Lefschetz's contributions on the topic is so weighty that his tract, even in unrevised form, is still an indispensable text for the serious student of the subject.

\section{Functional Operators}

By John von Neumann. Vol. 1: Measures and Integrals. (Annals of Mathematics Studies, No. 21.) Pp. vii +261 . (Princeton, N.J. : Princeton University Press; London: Oxford University Press, 1950.) 22s. 6d. net.

THE general mathematical theory of operators

has been much advanced by von Neumann's lectures at the Institute for Advanced Study, Princeton, now printed in the "Annals of Mathematics Studies". The first volume is almost entirely devoted to the theory of integration, which is given in a completeness probably beyond what will be required in the second and succeeding volumes.

About one-third of the book deals with the theory of Lebesgue measure and integration, in $n$-dimensional spaces, giving a concise and fairly comprehensive account of the basic concepts and theorems on the Lebesgue integral. The rest of the book is divided about equally between two chapters, on $\theta$ of which generalizes the measure theory to abstract spaces, and incidentally provides a definition of the Lebesgue integral independent of the topological ideas usually involved; the last chapter gives a thorough discussion of the general integral, and deals in detail with the derivative of one set function with respect to another.

The whole treatment is detailed, accurate and painstakingly thorough. It will, of course, appeal only to the pure mathematician with a taste for abstract spaces, but to such a reader the exposition will present no great difficulty. 